\title{
Facility Placement with Sub-Aisle Design in an Existing Layout
}

\author{
Min Zhang ${ }^{1}$, Selçuk Savas ${ }^{2}$, Rajan Batta $^{* 3}$, and Rakesh Nagi ${ }^{3}$ \\ ${ }^{1}$ Avis Budget Group Inc., 6 Sylvan Way, Parsippany, NJ 07054 \\ ${ }^{2}$ Department of Industrial Engineering, IŞIK University, Istanbul, Turkey \\ ${ }^{3}$ Department of Industrial and Systems Engineering, 438 Bell Hall, \\ University at Buffalo (SUNY), Buffalo, NY, 14260, USA
}

Final revision June 2008

\begin{abstract}
In this paper we consider the integration of facility placement in an existing layout and the configuration of one or two connecting sub-aisles. This is relevant, for example, when placing a new machine/department on a shop floor with existing machines/departments and an existing aisle structure. Our work is motivated by the work of Savas et al. (2002), that considered the optimal planar placement of a finite-size facility in the presence of existing facilities. Our work differs from theirs in that we consider material handling to be restricted to the aisle structure. We do not allow the newly placed facility to overlap with existing facilities or with the aisle structure. Facilities are rectangular and travel is limited to new or existing aisles. We show that there are a finite number of candidate placements for the new facility. Algorithms are developed to find the optimal placement and the corresponding configurations for the subaisles. Complexity of the solution method is analyzed. Also, a numerical example is provided to explore the impact of the number of sub-aisles added.
\end{abstract}

Keywords: Facility Design, Facility Placement, Sub-aisle Configuration.

\section{Introduction}

Facility layout and location problems arise in a variety of contexts (Francis et al. 1992). Facility layout problems consider placement of finite-sized facilities, while facility location problems consider placement of infinitesimal-sized facilities, perhaps in the presence of finite-sized barriers to travel or forbidden regions for locating new facilities. In facility layout and location problems, the material handling between facilities is assumed to take place along a shortest feasible rectilinear/Euclidean path. This assumption often leads to inaccurate distance measurements in a plant layout application, because material handling clearly has to take place through an aisle structure, i.e. travel has to be restricted to a material handling network. In network location problems, travel between facilities is assumed to occur through an underlying network, additionally both the existing facilities on the network and the new facilities to be located are assumed to be of infinitesimal

\footnotetext{
*To whom all correspondence should be addressed. E-mail: batta@eng.buffalo.edu
} 
size. The latter assumption is also not valid for plant layout problems. Recognizing the relevance of facility size and the existence of aisles for material handling between facilities in a plant, we address the layout addition problem of placing a rectangular new facility in a plane in the presence of other rectangular existing facilities and an aisle structure. We consider the cases of placing the new facility directly onto the existing aisles and adding one or two sub-aisles to connect the new facility to the existing aisles.

The rest of the paper is organized as follows. Section 2 provides a literature review. In Section 3 , we formally introduce and define the problem. In Section 4, we describe a grid construction procedure. In Section 5, we detail our approach to the problem, considering both the situation of adding a new facility directly onto the existing aisle structure, and the simultaneous consideration of placement of a new facility and configuration of sub-aisles. The complexity of our solution algorithm is analyzed in Section 6. An example is presented in Section 7. Finally, a summary and conclusions of our work are presented in Section 8.

\section{Literature Review}

Restricted location problems are the closely related research areas to our work where restrictions are imposed on locating new facilities. The restricted area falls into three classes: barriers (which prohibit location and travel), forbidden regions (which prohibit location but permit travel at no extra cost), and generalized congested regions, GCR (which prohibit location but permit travel at a penalty). For restricted location problems, readers are referred to Katz and Cooper (1981), Larson and Sadiq (1983), Batta et al. (1989), Aneja and Parlar (1994), Butt and Cavalier (1996), Klamroth (2000), Dearing et al. (2002) and Nandikonda et al. (2003), among others.

In all the studies referenced above, the authors assumed that the new facilities to be located were infinitesimal. Recognizing the practical relevance of facility size consideration, Savas et al. (2002) first considered the optimal placement of a finite-size new facility in the presence of arbitrarilyshaped barriers with the median objective and rectilinear distance metric. Variant works of Savas et al. (2002) have been studied by Sarkar et al. (2005), Kelachankuttu et al. (2007) and Sarkar et al. (2007). Sarkar et al. (2005) considered the placement of a finite-size new facility in the presence of existing facilities with a median objective, rectilinear distance metric, and rectangular shaped facilities. The restricted area is in the form of generalized congested regions as compared to barriers in Savas et al. (2002), additionally the area of the new facility to be located is known but its exact dimensions are to be determined, whereas the shape of the new facility is known $a$ priori in Savas et al. (2002). Kelachankuttu et al. (2007) developed contour lines with respect to placing a new rectangular facility in the presence of rectangular existing facilities. Their study is important when the optimal site is not suitable for facility placement with practical constraints. Sarkar et al. (2007) revisited the study of Savas et al. with a center objective. 
The above studies assumed that the material handling between facilities took place along a shortest feasible rectilinear path between the facility servers (I/O points). As claimed in the introduction, interaction between facilities is normally restricted to a material handling network in a plant layout application. A wide variety of network location problems have been studied, for example, $p$-median problem (Hakimi 1964), fixed charge location problem (Kuehn and Hamburger 1963), set covering location problem (Toregas et al. 1971), maximal covering location problem (Church and ReVelle 1979), etc. An excellent review of discrete network location problems can be found in Drezner and Hamacher (2004). In an attempt to relax the assumption that the underlying network is given, facility location-network design models were proposed, for example, Melkote (1996) proposed models to simultaneously optimize facility location and the underlying network design. Generally, facility location-network design models and network design models do not take into account the issue of the facility's size, which is of obvious importance in plant layout. This motivates the work presented in this paper.

Our work, in a way, may be also viewed as an extension of the work by Savas et al. (2002). We consider placing a new facility while using an existing aisle structure. As travel is restricted to the aisles, it may be necessary and/or advantageous to construct new sub-aisles which connect the new facility to the existing aisle structure. Motivated by these considerations we analyze the problem of placement of a finite-size new facility as well as connection of the new facility to the existing aisle structure with restricting travel on aisles.

\section{Problem Description}

\subsection{Problem Statement}

The problem that we address is that of placing a new facility (NF) in a manufacturing plant. This $\mathrm{NF}$ must be placed so that it does not overlap either the existing facilities (EFs) or the existing aisle structure. For the sake of analytical tractability we make several key simplifying assumptions:

- The plant is rectangular.

- The EFs are rectangular, with the sides of each EF parallel to the sides of the plant.

- There is an existing aisle structure through which all material handling takes place. The segments of this aisle structure are parallel to the sides of the plant.

- Each EF has a single I/O point which is located on the aisle structure.

- The NF is rectangular and placement must be done so that the sides of the NF are parallel to the sides of the plant.

- The NF has a single I/O point. 
The first version of the problem we consider is when no new aisles can be constructed. Thus the NF has to be placed so that its I/O point coincides with the existing aisle structure. In this case we focus on the objective of minimizing the material handling cost between the EFs and the NF.

The second version of the problem is when sub-aisle construction is permitted to connect the NF to the existing aisle structure. Both the cases of one new sub-aisle and two new sub-aisles are examined. Each newly constructed sub-aisle must be parallel to the sides of the plant, and it cannot overlap with the existing aisles except for exactly one connection point where the new subaisle intersects the existing aisle structure. The introduction of one or two sub-aisles can decrease the shortest distance between two EFs. Thus the objective in this case is minimization of the total material handling cost (i.e. between the EFs, and between the NF and EFs) plus the cost of constructing sub-aisles.

\subsection{Definitions and Notation}

We assume that each facility, including the new one, is a rectangular region of finite area in $\Re^{2}$. Let $B_{j}$ denote the set of points $(x, y) \in \Re^{2}$ that represent EF $j$. The collection of the EFs is represented by $B=\cup_{j} B_{j}$, and $D$ denotes the set of I/O points associated with the EFs. Let $H$ denote the set of points that represent the NF.

$G=(N, A)$ denotes the set of points in $\Re^{2}$ that represent the network of the existing aisle structure, where $N$ is the set of nodes including the intersections or end points of aisles and the I/O points associated with the EFs, and $A$ is the set of arcs denoting the aisles between a pair of nodes. An illustration of such a system is shown in Figure 1.a, where the bold lines represent the existing aisles and the black and white dots denote the nodes. Specifically, the black dots (nodes 1, 2 and 3) represent the I/O points of the EFs.

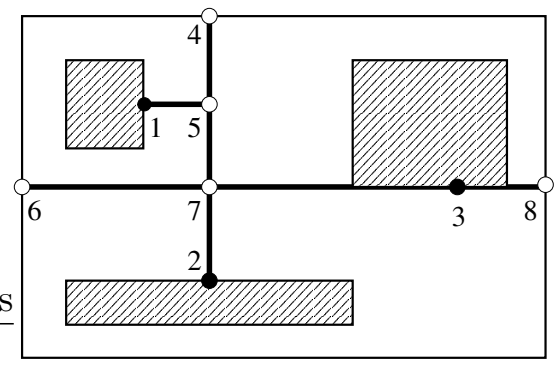

(a) the aisle network

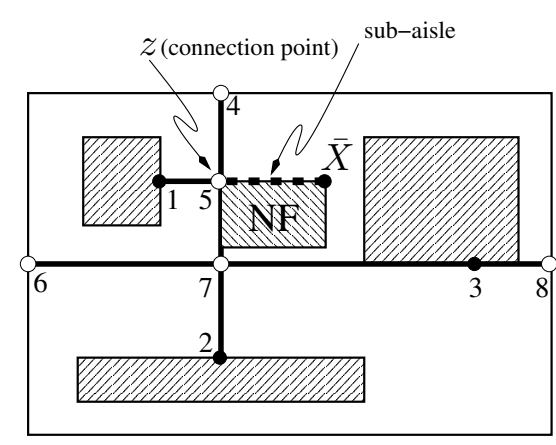

(b) placement of the NF and sub-aisles configurtation

Figure 1: Illustration of the system considered.

For the first version of the problem, the coordinates of the I/O point for the NF determine 
a solution. However, this is not the case for the second version of our problem. Each newly constructed sub-aisle intersects the existing aisle structure at a connection point. The optimal subaisle from a given location $\bar{X}$ to a connection point $z \in G$ has to be a shortest feasible rectilinear path between them. Consequently we let $\ell[\bar{X}, Z, P]$ denote the solution for our problem. Here $\bar{X}=(x, y)$ represents the location (point coordinates) for the NF's I/O point, $Z$ is the set of locations with its $i^{\text {th }}$ element $z_{i}=\left(x_{z_{i}}, y_{z_{i}}\right)$ representing the coordinates for the $i^{\text {th }}$ connection point, and $P$ is the set of paths with its $i^{t h}$ element $p_{i}$ denoting the shortest feasible rectilinear path from $\bar{X}$ to the $i^{\text {th }}$ connection point in $Z$. Figure 1.b illustrates the placement of the NF, one newly constructed sub-aisle (dash line) and a single connection point $z$.

Let $H(\bar{X})$ denote the set of points that correspond to the NF when its I/O point is at $\bar{X}$. The feasible region for the placement of the NF is defined as $F=\{\bar{X}: H(\bar{X}) \cap B=\phi, H(\bar{X}) \cap G=\phi\}$. Readers are referred to Savas et al. (2002) for details of identification of the feasible region.

There are two types of material handling interactions in our problem. First, there is interaction between $\mathrm{EF} i$ and the NF, the frequency of which is denoted by $w_{i} \geq 0$. Second, there is interaction between $\mathrm{EF} i$ and $\mathrm{EF} j$, the frequency of which is denoted by $h_{i j} \geq 0$. The interaction between any two points takes place through a shortest path on the aisle network. Consider a solution $\ell$, which indicates a feasible placement of the NF and one or more newly constructed sub-aisles that link the I/O point of the NF to the existing aisle structure. Let $d_{\ell}(i, j)$ represent the shortest path distance between the I/O points of EFs $i$ and $j$ when the solution is $\ell$, and let $d_{\ell}(i, \bar{X})$ represent the shortest path distance between the I/O points of EF $i$ and the NF at $\bar{X}$ given $\ell$. We also let $r_{\ell}$ denote the total length of constructed new sub-aisles for solution $\ell$.

For a given solution $\ell$, the total weighted travel distance between the NF and EFs is $f_{1}(\ell)=\sum_{i \in D} w_{i} d_{\ell}(i, \bar{X})$.

Similarly, the total weighted travel distance between the EFs is $f_{2}(\ell)=\sum_{i \in D} \sum_{j \in D} h_{i j} d_{\ell}(i, j)$.

The construction cost for the new sub-aisles is $f_{3}(\ell)=K r_{\ell}$,

where $K$ is the unit construction cost appropriately amortized with respect to units of $w_{i}$ and $h_{i j}$.

We seek a solution that minimizes the sum of $f_{1}(\ell), f_{2}(\ell)$ and $f_{3}(\ell)$.

\section{Preliminaries}

To facilitate the presentation of our approach, we describe the grid construction procedure that helps identify the basic cell for our analysis, introduce necessary definitions and present a few results from Hakimi (1964) and Savas et al. (2002). 


\subsection{Grid Construction and Cell Formation}

A grid construction procedure in the presence of barriers that divides the region into cells has been established in the work of Larson and Sadiq (1983); we note that the NF and EFs all act as barriers. We follow the same procedure to draw horizontal and vertical lines through the vertices of each EF and its I/O point, with each line terminated at the boundary of the first EF encountered, or at the boundary of the bounding rectangle that encloses all EFs and the existing aisle structure. We also draw horizontal and vertical lines through the nodes on the aisle network, with each line terminated at the boundary of the first EF encountered, or at the boundary of the bounding rectangle. We refer to these lines formed as gridlines. This grid is an extension of the grid proposed by Larson and Sadiq (1983) because additional lines are drawn with respect to the aisle network.

The EFs and existing aisle structure along with the gridlines divide the feasible region into a number of cells. All the cells generated are also rectangular with horizontal and vertical edges because the EFs are rectangular and the aisles are also horizontal or vertical. Each cell boundary is composed of segments of EFs, segments of aisles or gridlines. We note that the aisles are also divided into a set of segments, called edges by these gridlines. An illustration of gridlines constructed for a three-EF example is shown in Figure 2, where arc $(7,3)$ is divided into two edges $(7,9)$ and $(9,3)$.

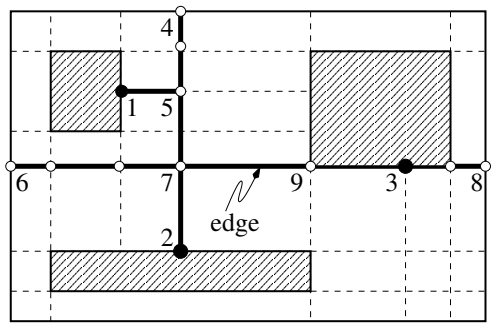

Figure 2: Illustration of grid construction.

The set of feasible placements within cell $C$ is denoted by $F_{C}$. It is pertinent to note here that a feasible placement $\bar{X} \in F_{C}$ does not necessarily require containment of the NF within cell $C$. $F_{C}$ is either an empty set, a segment, a rectangle or a combination of rectangles because the NF and cells are rectangles. On a horizontal/vertical segment $A B$ within cell $C$, if $\bar{X}$ is feasible at both $A$ and $B$ but not feasible on a location strictly within $A B$, there must exist an $\mathrm{EF}$ or an edge to cause this situation. In this case, cell $C$ would not exist according to the above grid construction procedure. Additionally, $\bar{X}$ is assumed to be on the boundary of the NF, consequently if $F_{C}$ is a combination of rectangles, it is of $\mathrm{L}$ shape or $\mathrm{T}$ shape as Figure 3 illustrates. Without loss of generality, $F_{C}$ is assumed to be of $\mathrm{T}$ shape and is divided into sub-rectangles as shown in Figure 3. Note that at most three sub-rectangles will be formed in $F_{C} . R\left(F_{C}\right)$ denotes one of the sub-rectangles in $F_{C}$. Let $E_{1}\left(R\left(F_{C}\right)\right), E_{2}\left(R\left(F_{C}\right)\right), E_{3}\left(R\left(F_{C}\right)\right)$ and $E_{4}\left(R\left(F_{C}\right)\right)$ denote the corners of $R\left(F_{C}\right)$, starting 
from the bottom left corner and labeling in the counter-clockwise direction. Let $C_{1}, C_{2}, C_{3}, C_{4}$ denote the corners of $C$, starting from the bottom left corner and labeling in the counter-clockwise direction. The grid construction procedure partitions the region outside a cell $C$ into at most eight sub-regions. For the sake of convenience, we give names to the sub-regions obtained with respect to this cell, as noted in Figure 4. Note that the sub-regions outside an cell do not include their boundaries.

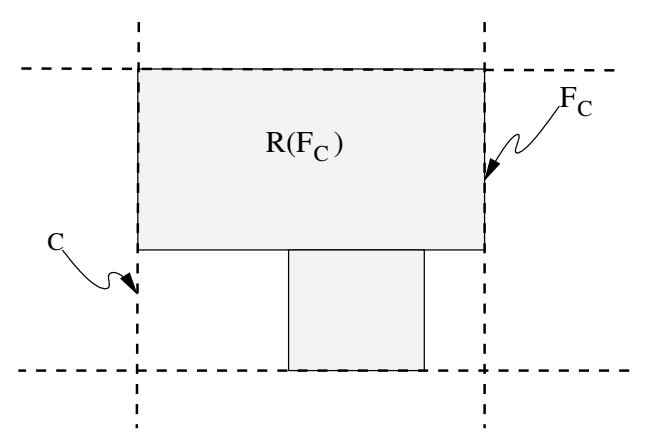

Figure 3: Sub-rectangles of feasible region within a cell.

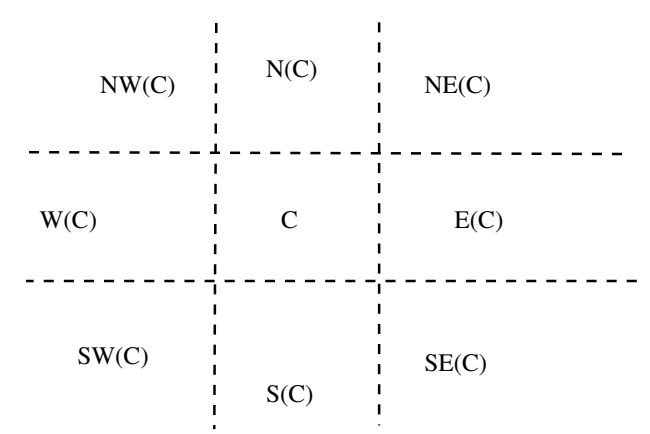

Figure 4: The eight sub-regions around a cell.

\subsection{An Edge and a Cell}

To facilitate the analysis in Section 5, we introduce the following definitions. Let $\left(x_{i}, y_{i}\right)$ be the coordinates of point $i$. Given an edge $(u, v)$ with coordinates $\left(x_{u}, y_{u}\right)$ and $\left(x_{v}, y_{v}\right)$, if $(u, v)$ is horizontal (vertical), we assume that $x_{u}<x_{v}\left(y_{u}<y_{v}\right)$.

Definition 1: Two points $i$ and $j$ with coordinates $\left(x_{i}, y_{i}\right)$ and $\left(x_{j}, y_{j}\right)$ are visible to each other if the shortest feasible rectilinear distance between the two points is $\left|x_{i}-x_{j}\right|+\left|y_{i}-y_{j}\right|$.

Definition 2: A point $i$ with coordinates $\left(x_{i}, y_{i}\right)$ is inside the span of a horizontal (vertical) edge $(u, v)$ with coordinates $\left(x_{u}, y_{u}\right)$ and $\left(x_{v}, y_{v}\right)$ if $x_{u} \leq x_{i} \leq x_{v}\left(y_{u} \leq y_{i} \leq y_{v}\right)$; otherwise point $i$ is outside the span of edge $(u, v)$.

Definition 3: A point $i$ with coordinates $\left(x_{i}, y_{i}\right)$ is visible to a horizontal edge $(u, v)$ if the shortest feasible rectilinear distance from $i$ to $(u, v)$ is equal to

$$
\begin{cases}\left|y_{i}-y_{u}\right| & \text { if } i \text { is inside the span of }(u, v), \\ \left|x_{i}-x_{u}\right|+\left|y_{i}-y_{u}\right| & \text { if } i \text { is to the left side of }(u, v), \\ \left|x_{i}-x_{v}\right|+\left|y_{i}-y_{v}\right| & \text { if } i \text { is to the right side of }(u, v) ;\end{cases}
$$

otherwise point $i$ is invisible to $(u, v)$. The visibility of point $i$ with respect to a vertical edge $(u, v)$ can be defined similarly.

Note that a point could be visible to an edge but outside the span of this edge. Also, a point could be inside the span of an edge but invisible to this edge. Figure 5 illustrates the possible cases.

Definition 4: A point $i$ with coordinates $\left(x_{i}, y_{i}\right)$ is inside the span of a cell $C$ if $X_{\min }(C) \leq$ $x_{i} \leq X_{\max }(C)$ or $Y_{\min }(C) \leq y_{i} \leq Y_{\max }(C)$, where $X_{\min }(C), X_{\max }(C), Y_{\min }(C), X_{\max }(C)$ are the respective bounds on $x$ coordinate and $y$ coordinate of the points inside cell $C$. 


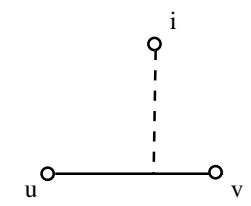

(a) visible and inside

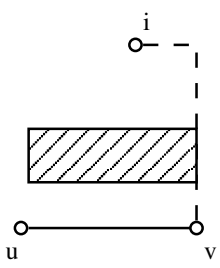

(c) invisible and inside

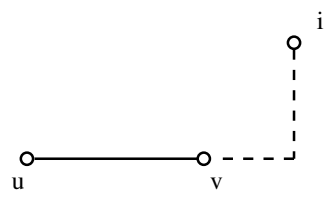

(b) visible and outside

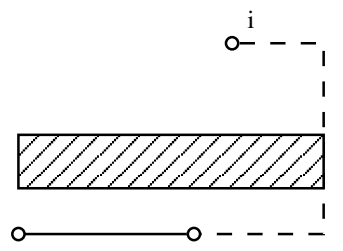

(d) invisible and outside

Figure 5: A point and an edge.

Definition 5: An edge $(u, v)$ is inside the span of a cell $C$ if any point on edge $(u, v)$ is inside the span of cell $C$; otherwise it is outside the span of $C$.

Definition 6: An edge $(u, v)$ is visible to a cell $C$ if any point inside $C$ is visible to $(u, v)$.

Note that an edge could be visible to a cell but outside the span of this cell. Also, an edge could be inside the span of a cell but invisible to this cell. Figure 6 illustrates the possible cases.

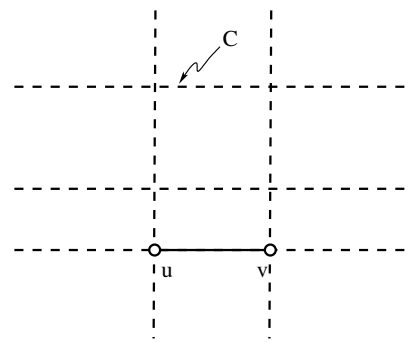

(a) visible and inside

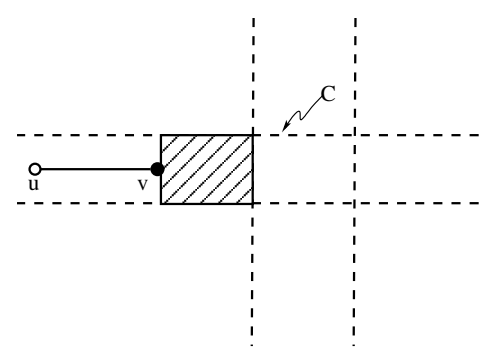

(c) invisible and inside

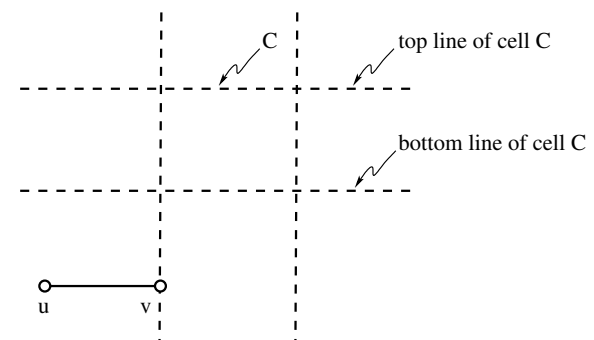

(b) visible and outside

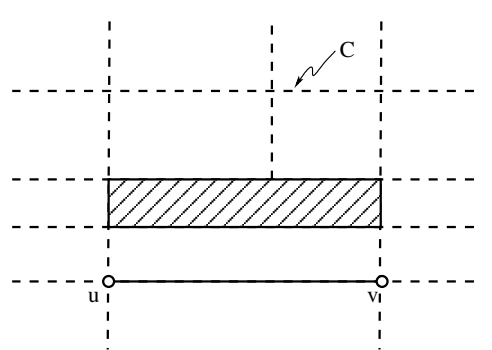

(d) invisible and outside

Figure 6: An edge and a cell.

Several other observations are in order: 
(1) An edge is either horizontal or vertical. Without loss of generality (since the coordinate system can be rotated by $90^{\circ}$ ), we assume that an edge is horizontal in this paper unless specified otherwise.

(2) An edge, which is fully contained in either one of the regions: $\mathrm{W}(C), \mathrm{S}(C), \mathrm{E}(C)$ and $\mathrm{N}(C)$, is inside the span of cell $C$. For example, edge $(u, v)$ in Figure 6.a and 6.c.

(3) An edge, which is fully contained in either one of the regions: $\mathrm{SW}(C), \operatorname{SE}(C), \mathrm{NE}(C)$ and $\mathrm{NW}(C)$, is outside the span of cell $C$. For example, edge $(u, v)$ in Figure 6.b.

(4) An edge in region $\mathrm{W}(C)$ or $\mathrm{E}(C)$ must be invisible to cell $C$, otherwise, cell $C$ would not exist. For example, edge $(u, v)$ in Figure 6.c.

(5) Edge $(u, v)$ may span several sub-regions above the top line of cell $C$, or those below the bottom line of cell $C$ as shown in Figure 6.d. In this case, edge $(u, v)$ must be invisible to cell $C$, otherwise, edge $(u, v)$ would not exist.

To this end, we conclude that four cases are sufficient for analysis: (1) Edge $(u, v)$ is fully contained inside sub-region $\mathrm{S}(C)$. (2) Edge $(u, v)$ is fully contained inside sub-region $\mathrm{SW}(C)$. (3) Edge $(u, v)$ is fully contained inside sub-region $\mathrm{W}(C)$. (4) Edge $(u, v)$ may span several sub-regions below the bottom line of $C$. This is because the results, if any for other cases, will follow directly due to symmetry. Figure 6 illustrates one example for each case.

\subsection{Some Results}

For our future analysis, it is necessary to list some previous results suitably adapted for our work. Result 1: Nodes on a network are candidate locations for $p$ NFs on the network so as to minimize the sum of weighted distance between the NFs and EFs located at nodes of the network. Result 1 is also referred as the nodal optimality property for the $p$-median problem on a network (Hakimi 1964).

Result 2: A shortest feasible rectilinear path which passes through cell corner $C_{k}$ and corner $E_{k}\left(R\left(F_{C}\right)\right), k \in\{1,2,3,4\}$ exists from the I/O point of each EF to the I/O point of NF at $\bar{X}$ for all $\bar{X} \in R\left(F_{C}\right)$ (Savas et al. 2002, Lemma 2).

\section{The Approach}

In this section, we present the approach for the two versions of our problem. We start by considering the situation where the NF is placed such that no new sub-aisle needs to be constructed. Then we consider the cases of constructing either one or two sub-aisles which connect the NF to the existing aisle structure. Note that the single sub-aisle case does not change the distance between the EFs, while the two sub-aisles case can. This significantly complicates the analysis. 


\subsection{Addition of One New Facility on to the Existing Aisle Structure}

We start with a discussion of the case of a single NF and no new sub-aisle. Here the problem is to find the best placement for the NF such that $\bar{X} \in G \cap F$. In this case, it is obvious that $f_{2}(\ell)$ is a constant and $f_{3}(\ell)$ is equal to 0 . Therefore we want to minimize $f_{1}(\ell)$.

The problem is similar to the 1-median location problem (Hakimi 1964), except that the placement of $\bar{X}$ has to be feasible, i.e. the resultant NF will not overlap with the EFs or interfere with the existing aisle structure. If the Hakimi node is a feasible location, then it is obviously the optimal location. However, the Hakimi node may not always be feasible. For instance, suppose that node 2 in Figure 1 happens to be the Hakimi node. This location may not be feasible as it may cause an overlap between the NF and the existing aisles. To this end, we define a segment $[p, q] \subset G$ as a feasible segment if $\bar{X}$ is feasible over $[p, q]$.

Theorem 1 For any feasible segment $[p, q] \subset G$, the function $f_{1}(\ell)$ is concave over $[p, q]$.

Proof: This is a corollary of the main nodal optimality result in Hakimi (1964).

Let $Q$ denote the set of end points of all the feasible segments. Theorem 1 implies that $Q$ is the set of candidate locations for the optimal placement of the NF on the existing aisle structure. The element of the set $Q$ with minimum value of $f_{1}(\ell)$ is an optimal placement of $\bar{X}$.

\subsection{New Facility Placement and Configuration of a Single Sub-Aisle}

We now allow free placement of the NF as long as there is no interference between the NF and the EFs or the aisle structure. We construct one sub-aisle that connects the NF to the existing aisle structure. This sub-aisle cannot interfere with any of the EFs nor overlap with the existing aisle structure. The problem, in this case, is similar to the 1-median problem as the construction of one sub-aisle would not affect the interactions between the EFs. Consequently, we want to minimize $f_{1}(\ell)+f_{3}(\ell)$, i.e.,

$$
\min _{\bar{X} \in F} \min _{z \in G}\left\{\sum_{i \in D} w_{i}[d(i, z)+r(z, \bar{X})]+K r(z, \bar{X})\right\} .
$$

Here $d(i, z)$ denotes the distance of the shortest path on the aisle network which connects EF $i$ and $z$, and $r(z, \bar{X})$ denotes the distance of a shortest feasible rectilinear path between $z$ and $\bar{X}$.

We now present Lemma 1, which then provides the basis for an algorithm for determining the optimal solution.

Lemma 1 Given a location $\bar{X}$ and an edge $(u, v)$, the optimal sub-aisle between $\bar{X}$ and $(u, v)$ that minimizes $f_{1}(\ell)+f_{3}(\ell)$ is the shortest feasible rectilinear path between them. 
Proof: Consider the edge $(u, v)$ in Figure 7. Let $z$ denote the shortest connection point from $\bar{X}$ to $(u, v)$ and let $z^{\prime}$ denote any connection point such that $r\left(z^{\prime}, \bar{X}\right)>r(z, \bar{X})$. We need to prove that $f_{1}\left(\bar{X}, z^{\prime}\right)+f_{3}\left(\bar{X}, z^{\prime}\right)>f_{1}(\bar{X}, z)+f_{3}(\bar{X}, z)$.

It is obvious that $f_{3}\left(\bar{X}, z^{\prime}\right)>f_{3}(\bar{X}, z)$.

The following proof shows $f_{1}\left(\bar{X}, z^{\prime}\right) \geq f_{1}(\bar{X}, z)$, using similar arguments to those in Hakimi (1964). $N_{1}$ is defined as the set of nodes that reaches $\bar{X}$ more efficiently through node $u$ and $N_{2}$ as the set of nodes reaching $\bar{X}$ more efficiently through $v$, with ties broken arbitrarily. $N_{1}$ and $N_{2}$ are thus mutually exclusive sets whose union is equal to $D$. If the sub-aisle aisle changes from $(\bar{X}, z)$ to $\left(\bar{X}, z^{\prime}\right)$, nodes in $N_{1}$ require a longer distance to reach $\bar{X}$ through $u$. For those nodes in $N_{2}$, the distance to $\bar{X}$ through $v$ remains unchanged. The result follows.

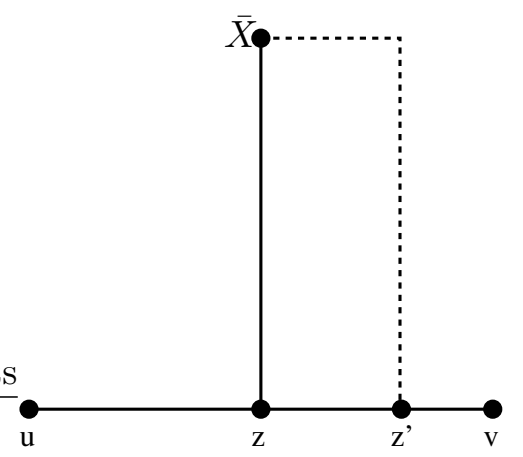

Figure 7: Sub-aisle connection to an existing edge.

Lemma 1 implies that the best configuration of a single sub-aisle uses the shortest feasible rectilinear path between the location of the NF and an edge that need to be connected. Note that there possibly exist multiple shortest feasible rectilinear paths from a location to an edge, in other words, multiple optimal configurations of a single sub-aisle may exist. In this case, one of the shortest feasible rectilinear paths can be determined using an algorithm similar to the one in Larson and $\mathrm{Li} \mathrm{(1981).} \mathrm{In} \mathrm{the} \mathrm{following} \mathrm{sections} \mathrm{of} \mathrm{the} \mathrm{paper,} \mathrm{the} \mathrm{best} \mathrm{configuration} \mathrm{of} \mathrm{a} \mathrm{single} \mathrm{sub-aisle} \mathrm{is}$ defined as the shortest feasible rectilinear path determined by using such an algorithm. According to Lemma 1, Algorithm 1 in the following chooses the best solution from all the combinations of a NF's location and an edge.

\section{Algorithm 1 (Single New Facility, One Sub-aisle Case)}

1. FOR each cell $C \subset F$

2. IF $F_{C} \neq \phi$ THEN

3. For each $R\left(F_{C}\right) \subset F_{C}$

4. $\quad$ FOR each edge $(u, v) \in A$ 
5.

6.

7.
FOR each candidate location $\bar{X} \in R\left(F_{C}\right)$

Determine the shortest feasible rectilinear path from $\bar{X}$ to $(u, v)$

Evaluate the objective function and record the best solution

Algorithm 1 will achieve the optimal solution within finite steps only if there are a finite number of candidate locations to be considered in each $R\left(F_{C}\right) \subset F_{C}$. Lemma 2 below shows that at most two candidate placements in each $R\left(F_{C}\right) \subset F_{C}$ with respect to edge $(u, v)$ need to be considered.

Lemma 2 Given an edge $(u, v)$ and a cell $C$, at most two candidate placements with respect to edge $(u, v)$ for the $N F$ in each $R\left(F_{C}\right) \subset F_{C}$ need to be considered and the candidate placement is one of the corners of $R\left(F_{C}\right)$.

\section{Proof:}

(1) We first start with the case where edge $(u, v)$ is inside the span of cell $C$ and also visible to $C$, as Figure 8 illustrates. The NF and $R\left(F_{C}\right)$ are shown in Figure 8. It is obvious that any placement inside $R\left(F_{C}\right)$ is dominated by at least one placement on segment $\left[E_{1}\left(R\left(F_{C}\right)\right), E_{2}\left(R\left(F_{C}\right)\right)\right]$, which is the closest side to $(u, v)$. According to the main nodal optimality result in Hakimi (1964), it is not difficult to show that the objective function $f_{1}(\bar{X}, z)+f_{3}(\bar{X}, z)$ is concave over segment $\left[E_{1}\left(R\left(F_{C}\right)\right), E_{2}\left(R\left(F_{C}\right)\right)\right]$. Thus the candidate placements should be $E_{1}\left(R\left(F_{C}\right)\right)$ and $E_{2}\left(R\left(F_{C}\right)\right)$. In general, the two corners on the side of $R\left(F_{C}\right)$, which is parallel and closer to $(u, v)$, are the candidate placements. This establishes the result for this specific situation.

(2) Many other cases are possible as illustrated in Figure 6. In these cases, we first prove the following statements: (a) a shortest feasible rectilinear path from any placement at $\bar{X} \in R\left(F_{C}\right)$ to edge $(u, v)$ will intersect edge $(u, v)$ either at $u$ or $v$, i.e. one of the end nodes of $(u, v)$; (b) a shortest feasible rectilinear path which passes through $C_{k}$ and $E_{k}\left(R\left(F_{C}\right)\right), k \in\{1,2,3,4\}$, exists from $\bar{X} \in R\left(F_{C}\right)$ to $u(v)$. We then demonstrate that Lemma 2 holds according to statements (a) and $(b)$.

For the case where edge $(u, v)$ is outside the span of $C$ and visible to $C$ (Figure 6.b), it is obvious that the shortest feasible rectilinear path from $\bar{X} \in R\left(F_{C}\right)$ to $(u, v)$ must intersect $(u, v)$ at $u$ or $v$. If edge $(u, v)$ is invisible to $C$, it could be inside or outside the span of $C$ (see Figures 6.c and 6.d). The same argument is employed to prove these two sub-cases. Suppose that the shortest feasible rectilinear path from $\bar{X} \in R\left(F_{C}\right)$ to edge $(u, v)$ intersects $(u, v)$ at $z$ instead of $u$ or $v$, there must exist at least one EF (acting as a barrier) to drive the shortest feasible rectilinear path from $\bar{X} \in R\left(F_{C}\right)$ to $(u, v)$ to arrive at $z$, as shown in Figure 9 . In this case, edge $(u, v)$ would not exist according to the grid construction procedure in Section 3.1. 
Therefore statement (a) follows. According to Result 2, a shortest feasible rectilinear path which passes through cell corner $C_{k}$ and corner $E_{k}\left(R\left(F_{C}\right)\right), k \in\{1,2,3,4\}$ exists from $u(v)$ to $\bar{X}$ $\in R\left(F_{C}\right)$. Statement (b) follows.

Statements (a) and (b) together show that any placement inside $R\left(F_{C}\right)$ is dominated by one of the corners $E_{k}\left(R\left(F_{C}\right)\right), k \in\{1,2,3,4\}$. This is because $r(\bar{X}, u) \geq \min _{k \in\{1,2,3,4\}}\left\{r\left(E_{k}\left(R\left(F_{C}\right)\right), u\right)\right\}$ or $r(\bar{X}, v) \geq \min _{k \in\{1,2,3,4\}}\left\{r\left(E_{k}\left(R\left(F_{C}\right)\right), v\right)\right\}$. Therefore we let $E_{u}$ denote the set of corners such that the corresponding shortest feasible rectilinear paths share node $u$ as a connection point. Similarly, $E_{v}$ is defined as the set of corners such that these paths share node $v$ as a connection point. Let $r\left(E_{k}\left(R\left(F_{C}\right)\right),(u, v)\right)$ denote the shortest feasible rectilinear distance from corner $E_{k}\left(R\left(F_{C}\right)\right), k \in\{1,2,3,4\}$ to edge $(u, v)$. Let $\hat{k}_{u}=\operatorname{argmin}\left\{r\left(E_{k}\left(R\left(F_{C}\right)\right),(u, v)\right), k \in E_{u}\right\}$ and $\hat{k}_{v}=\operatorname{argmin}\left\{r\left(E_{k}\left(R\left(F_{C}\right)\right),(u, v)\right), k \in E_{v}\right\}$, then $E_{\hat{k}_{u}}\left(R\left(F_{C}\right)\right)$ and $E_{\hat{k}_{v}}\left(R\left(F_{C}\right)\right)$ are the candidate placements.
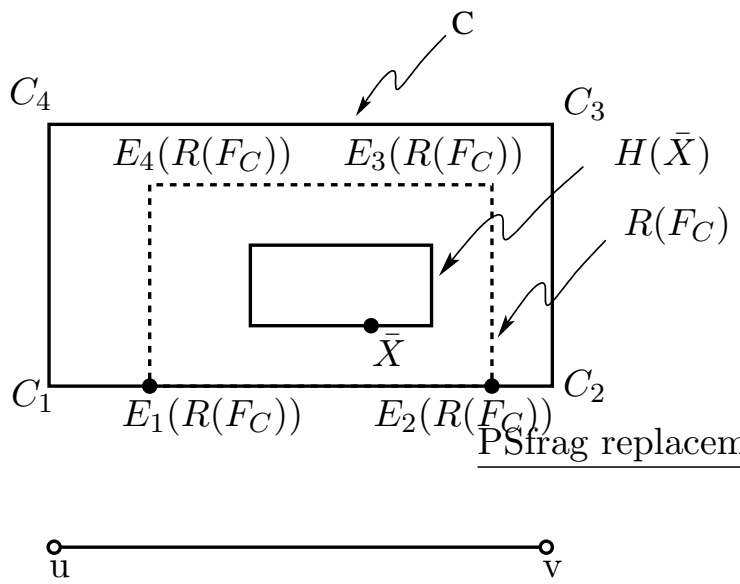

Figure 8: Candidate placements with respect to an edge.

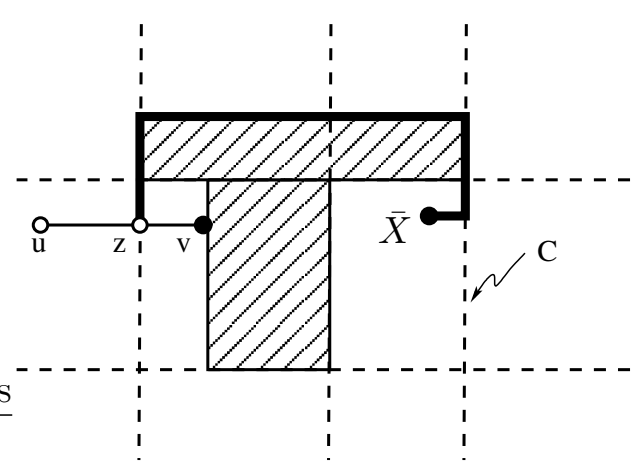

Figure 9: Connection at end nodes of an edge.

Lemma 2 permits us to arrive at the following result.

Theorem 2 There are a finite number of candidate placements for the NF and a finite number of candidate configurations of one sub-aisle that need to be considered when seeking an optimal solution for placement of NF and construction of one sub-aisle.

Proof: The feasible region for the placement of the NF is divided into a finite number of cells. Also, there are a finite number of edges in the existing aisle structure. Thus the number of combinations of cell/edge is finite. According to Lemma 2 and the fact that at most three sub-rectangles can be formed in each cell, each cell/edge combination considers at most six candidate placements for the NF. Since the best sub-aisle configuration from one location to one edge is defined as the shortest feasible rectilinear path between them determined by an algorithm similar to the one in Larson and $\mathrm{Li}$ (1981), the number of candidate sub-aisle configurations is also finite. The result follows. 


\subsection{Placement of One New Facility and Configuration of Two Sub-Aisles}

We now consider the case of constructing two sub-aisles from the NF to the existing aisle structure. For this case we also need to take into consideration the material handling costs between the EFs, since the addition of two new sub-aisles may create a shorter alternate path for some of the existing flows. The two new sub-aisles intersect the existing aisle structure at two connection points (denoted by $z_{1}$ and $z_{2}$ ), $f_{1}(\ell), f_{2}(\ell)$ and $f_{3}(\ell)$ are rewritten as follows:

$$
\begin{aligned}
& f_{1}(\ell)=f_{1}\left(\bar{X}, z_{1}, z_{2}\right)=\sum_{i \in D} w_{i} \min \left\{d\left(i, z_{1}\right)+r\left(z_{1}, \bar{X}\right), d\left(i, z_{2}\right)+r\left(z_{2}, \bar{X}\right)\right\}, \\
& f_{2}(\ell)=f_{2}\left(\bar{X}, z_{1}, z_{2}\right)=\sum_{i \in D} \sum_{j \in D} h_{i j} d_{\ell}(i, j), \text { and } \\
& f_{3}(\ell)=f_{3}\left(\bar{X}, z_{1}, z_{2}\right)=K\left[r\left(z_{1}, \bar{X}\right)+r\left(z_{2}, \bar{X}\right)\right] .
\end{aligned}
$$

where the subscript $\ell$ signifies that the shortest distance between $\mathrm{EF} i$ and $\mathrm{EF} j$ may change due to the two newly constructed sub-aisles.

Let $f\left(\bar{X}, z_{1}, z_{2}\right)=f_{1}\left(\bar{X}, z_{1}, z_{2}\right)+f_{2}\left(\bar{X}, z_{1}, z_{2}\right)+f_{3}\left(\bar{X}, z_{1}, z_{2}\right)$. The optimization problem can be restated as follows:

$$
\min _{\bar{X} \in F} \min _{z_{1}, z_{2} \in G}\left\{f_{1}\left(\bar{X}, z_{1}, z_{2}\right)+f_{2}\left(\bar{X}, z_{1}, z_{2}\right)+f_{3}\left(\bar{X}, z_{1}, z_{2}\right)\right\} .
$$

Lemma 3 presents a result similar to that in Lemma 1.

Lemma 3 Given a location $\bar{X}$, the optimal sub-aisles from $\bar{X}$ to two given separate edges consist of two shortest feasible rectilinear paths from $\bar{X}$ to each edge.

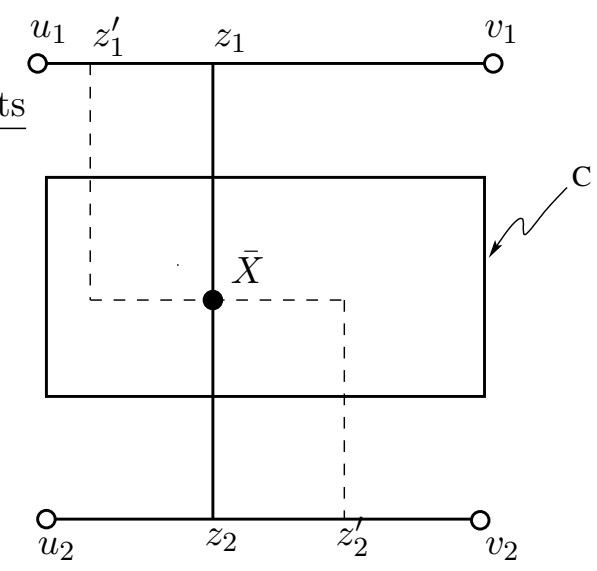

Figure 10: Shortest path connection to sub-aisles.

Proof: Consider edges $\left(u_{1}, v_{1}\right)$ and $\left(u_{2}, v_{2}\right)$ in Figure 10 where both edges are horizontal. Let $z_{1}$ and $z_{2}$ denote the shortest connection points from $\bar{X}$ to edge $\left(u_{1}, v_{1}\right)$ and $\left(u_{2}, v_{2}\right)$, respectively, and let $z_{1}^{\prime}, z_{2}^{\prime}$ denote any connection points such that $r\left(z_{1}^{\prime}, \bar{X}\right)>r\left(z_{1}, \bar{X}\right)$ and $r\left(z_{2}^{\prime}, \bar{X}\right)>r\left(z_{2}, \bar{X}\right)$. It is obvious that $f_{3}\left(\bar{X}, z_{1}^{\prime}, z_{2}^{\prime}\right)>f_{3}\left(\bar{X}, z_{1}, z_{2}\right)$. 
Similarly, $N_{1}, N_{2}, N_{3}$ and $N_{4}$ are defined as the sets of nodes that reach $\bar{X}$ most efficiently through $u_{1}, v_{1}, u_{2}$ and $v_{2}$, respectively (with ties broken arbitrarily). Following the logic presented in the proof of Lemma 1 , it is easy to show that $f_{1}\left(\bar{X}, z_{1}^{\prime}, z_{2}^{\prime}\right) \geq f_{1}\left(\bar{X}, z_{1}, z_{2}\right)$.

Again, applying the same logic, we have $f_{2}\left(\bar{X}, z_{1}^{\prime}, z_{2}^{\prime}\right) \geq f_{2}\left(\bar{X}, z_{1}, z_{2}\right)$.

The above demonstrates the result in the case where both edges are horizontal. Following the same logic, the result can also be established for the case where one edge is horizontal and one edge is vertical. Lemma 3 follows.

Lemma 3 implies that an algorithm similar to Algorithm 1 can be developed to evaluate all the combinations of a candidate locations for the NF and two edges. Therefore we first show in Lemma 4 that the candidate locations in $R\left(F_{C}\right)$ with respect to two edges are finite in a special case, then extend this result for a general case in Theorem 3. Finally, Algorithm 2 is presented for determining the optimal solution for the two sub-aisles case.

Before stating Lemma 4 , we introduce $\left.f\left(\bar{X}, z_{1}, z_{2}\right)\right|_{e_{1}, e_{2}}$ where the conditional subscript on $e_{1}$ and $e_{2}$ signifies the condition of being given $z_{1} \in e_{1}$ and $z_{2} \in e_{2}$.

Lemma 4 Consider two separate edges $e_{1}, e_{2}$ and a cell $C$, with the property that both edges are visible to $C$, as shown in Figure 11. The function $\left.f\left(\bar{X}, z_{1}, z_{2}\right)\right|_{e_{1}, e_{2}}$ is concave over the set $R\left(F_{C}\right)$.

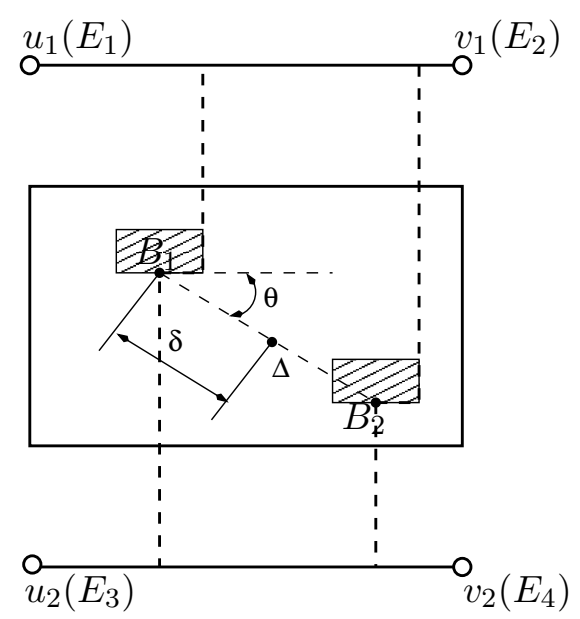

Figure 11: Movement of the NF within a cell.

Proof: We start with the case where both edges are horizontal. In the case where one edge is horizontal and one edge is vertical, the result can be demonstrated with the same argument. The following proof refers to Figure 11.

Let $e_{1}, e_{2}$ denote edges $\left(u_{1}, v_{1}\right)$ and $\left(u_{2}, v_{2}\right)$ respectively. We have $\left.f\left(\bar{X}, z_{1}, z_{2}\right)\right|_{e_{1}, e_{2}}=\left.f_{1}\left(\bar{X}, z_{1}, z_{2}\right)\right|_{e_{1}, e_{2}}+\left.f_{2}\left(\bar{X}, z_{1}, z_{2}\right)\right|_{e_{1}, e_{2}}+\left.f_{3}\left(\bar{X}, z_{1}, z_{2}\right)\right|_{e_{1}, e_{2}}$.

(1) We first prove that $\left.f_{1}\left(\bar{X}, z_{1}, z_{2}\right)\right|_{e_{1}, e_{2}}$ is concave over $R\left(F_{C}\right)$. 
Consider a location $B_{1} \in R\left(F_{C}\right)$. Further consider some feasible direction of movement from location $B_{1}$, as shown in Figure 11, to a location $B_{2}$, where $B_{2} \in R\left(F_{C}\right)$. Also any location $\Delta$ on the line segment joining $B_{1}$ and $B_{2}$ has the property $\Delta \in R\left(F_{C}\right)$ (If no such point $B_{2}$ exists, then $R\left(F_{C}\right)$ is a singleton set and the result follows).

Let the variable $\delta$ parametrize the movement along $\overline{B_{1} B_{2}}$ which is $\delta$ Euclidean distance units from $B_{1}$. For the sake of notation simplicity, let $E_{1}, E_{2}, E_{3}$ and $E_{4}$ denote $u_{1}, v_{1}, u_{2}$ and $v_{2}$ respectively, as shown in Figure 11. We have

$$
\left.f_{1}\left(\Delta, z_{1}, z_{2}\right)\right|_{e_{1}, e_{2}}=\sum_{i \in D} w_{i} \min _{k}\left\{d\left(i, E_{k}\right)+d\left(E_{k}, \Delta\right)\right\} .
$$

For given $k, d\left(i, E_{k}\right)$ is constant with respect to $\delta$, and it is easy to show that $d\left(E_{k}, \Delta\right)$ is linear in $\delta$ :

$$
d\left(E_{k}, \Delta\right)= \begin{cases}d\left(E_{k}, B_{1}\right)+\delta(+\cos \theta+\sin \theta) & \text { for } k=1, \\ d\left(E_{k}, B_{1}\right)+\delta(-\cos \theta+\sin \theta) & \text { for } k=2, \\ d\left(E_{k}, B_{1}\right)+\delta(+\cos \theta-\sin \theta) & \text { for } k=3, \\ d\left(E_{k}, B_{1}\right)+\delta(-\cos \theta-\sin \theta) & \text { for } k=4,\end{cases}
$$

where $\theta$ is as shown in Figure 11.

Therefore $d(i, \Delta)=\min _{k}\left\{d\left(i, E_{k}\right)+d\left(E_{k}, \Delta\right)\right\}$ is the minimum of four linear functions and is concave. Because $\left.f_{1}\left(\Delta, z_{1}, z_{2}\right)\right|_{e_{1}, e_{2}}$ is the sum of positively weighted concave functions, it is also concave.

(2) We now prove that $\left.f_{2}\left(\Delta, z_{1}, z_{2}\right)\right|_{e_{1}, e_{2}}$ is also concave over $R\left(F_{C}\right)$.

The two new sub-aisles may create a shorter alternate path for some of the existing flows. Therefore

$\left.f_{2}\left(\Delta, z_{1}, z_{2}\right)\right|_{e_{1}, e_{2}}=\sum_{i \in D} \sum_{j \in D} h_{i j} \min \left\{d(i, j), d_{\Delta}\left(i, E_{1}, E_{3}, j\right), d_{\Delta}\left(i, E_{1}, E_{4}, j\right), d_{\Delta}\left(i, E_{2}, E_{3}, j\right)\right.$, $\left.d_{\Delta}\left(i, E_{2}, E_{4}, j\right)\right\}$,

where $d_{\Delta}\left(i, E_{k}, E_{l}, j\right)=d\left(i, E_{k}\right)+d\left(E_{k}, \Delta\right)+d\left(\Delta, E_{l}\right)+d\left(E_{l}, j\right), k, l=1,2,3,4$.

$d(i, j), d\left(i, E_{k}\right)$ and $d\left(E_{l}, j\right)$ are constants with respect to $\delta$, and it is easy to show that $d\left(E_{k}, \Delta\right)+$ $d\left(\Delta, E_{l}\right)$ is linear in $\delta$.

We have

$$
d\left(E_{k}, \Delta\right)+d\left(\Delta, E_{l}\right)= \begin{cases}d\left(E_{k}, B_{1}\right)+d\left(B_{1}, E_{l}\right)+\delta(+2 \cos \theta) & \text { for } k=1, l=3, \\ d\left(E_{k}, B_{1}\right)+d\left(B_{1}, E_{l}\right) & \text { for } k=1, l=4, \\ d\left(E_{k}, B_{1}\right)+d\left(B_{1}, E_{l}\right) & \text { for } k=2, l=3, \\ d\left(E_{k}, B_{1}\right)+d\left(B_{1}, E_{l}\right)+\delta(-2 \sin \theta) & \text { for } k=2, l=4 .\end{cases}
$$

Therefore $D_{\Delta}(i, j)=\min \left\{d(i, j), d_{\Delta}\left(i, E_{1}, E_{3}, j\right), d_{\Delta}\left(i, E_{1}, E_{4}, j\right), d_{\Delta}\left(i, E_{2}, E_{3}, j\right), d_{\Delta}\left(i, E_{2}, E_{4}, j\right)\right\}$ is the minimum of five linear functions and therefore concave. Because $\left.f_{2}\left(\Delta, z_{1}, z_{2}\right)\right|_{e_{1}, e_{2}}$ is the sum of positively weighted concave functions, it is also concave.

(3) Since $\left.f_{3}\left(\Delta, z_{1}, z_{2}\right)\right|_{e_{1}, e_{2}}=\left.f_{3}\left(B_{1}, z_{1}, z_{2}\right)\right|_{e_{1}, e_{2}},\left.f_{3}\left(\Delta, z_{1}, z_{2}\right)\right|_{e_{1}, e_{2}}$ is constant with respect to $\delta$ and therefore concave.

We conclude that $\left.f\left(\bar{X}, z_{1}, z_{2}\right)\right|_{e_{1}, e_{2}}$ is concave over $R\left(F_{C}\right)$.

It is probable that an edge is invisible to a cell as Figure 12 illustrates. This case complicates the analysis. We now introduce several definitions similar to those defined in Nandikonda et al. 
(2003). A shortest feasible rectilinear path from $\bar{X} \in R\left(F_{C}\right)$ to edge $(u, v)$ passes through either one of the cell corners if $(u, v)$ is outside or invisible to cell $C$. We call that the assignment of edge $(u, v)$ to cell corner $C_{k}$, if this shortest feasible rectilinear path passes through $C_{k}, k=1,2,3,4$. If the shortest feasible rectilinear path from $\bar{X} \in R\left(F_{C}\right)$ to edge $(u, v)$ passes through $C_{k}$, its distance function can be explicitly presented as $r(\bar{X},(u, v))=d\left(\bar{X}, C_{k}\right)+r\left(C_{k},(u, v)\right)$, where $r\left(C_{k},(u, v)\right)$ is a constant.

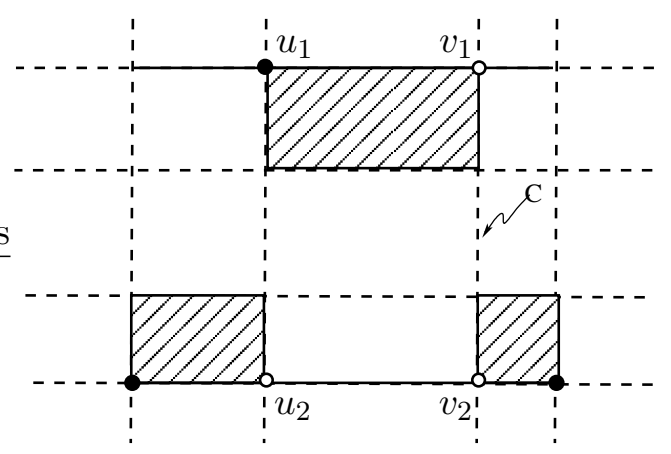

Figure 12: Edge $\left(u_{1}, v_{1}\right)$ invisible to cell $C$.

However, the assignment of edges to cell corners can change upon moving the placement $\bar{X}$ in $R\left(F_{C}\right)$, as illustrated by the example in Figure 13. When the placement $\bar{X} \in R\left(F_{C}\right)$ is to the left side of, but not on line segment $\overline{C_{5} C_{6}}$, a shortest feasible rectilinear path from $\bar{X}$ to $(u, v)$ goes through cell corner $C_{1}$. While if $\bar{X} \in R\left(F_{C}\right)$ is to the right side of, but not on line segment $\overline{C_{5} C_{6}}$, the path goes through cell corner $C_{2}$. For $\bar{X} \in R\left(F_{C}\right)$ on line segment $\overline{C_{5} C_{6}}$, both alternatives (going through $C_{1}$ or $C_{2}$ ) are equally attractive. For this reason, we call the line segment $\overline{C_{5} C_{6}}$ an Equal Travel Time Line (ETTL). Similar definitions can be found in Nandikonda et al. (2003).

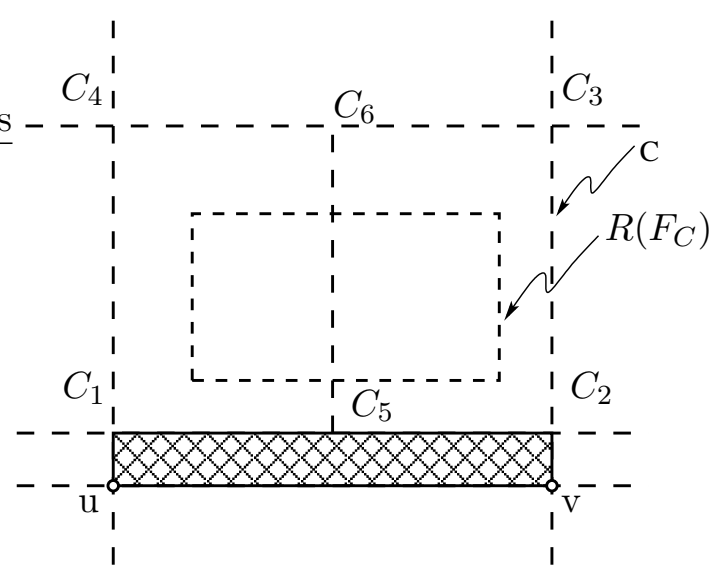

Figure 13: Example for varying cell corner assignment to edge.

We follow the procedure established in Nandikonda et al. (2003) to construct ETTLs associated 
with an edge $(u, v)$ and a cell $C$. Note that the edge is outside the span of the cell or invisible to it, otherwise the shortest feasible rectilinear path from $\bar{X} \in R\left(F_{C}\right)$ to $(u, v)$ may not pass through the cell corners as shown in Figure 5.a. In fact, an edge will generate at most one ETTL in a cell - see Nandikonda et al. (2003).

The distinctive feature of a sub-cell of $R\left(F_{C}\right)$ is that the form of the distance function $r(\bar{X},(u, v))$ does not change as long as $\bar{X}$ is within a sub-cell of $R\left(F_{C}\right)$. This is because for any $\bar{X}$ within a sub-cell of $R\left(F_{C}\right)$, a shortest feasible rectilinear path always goes though the same corner of $C$. Combining this observation and Lemma 4, we arrive at the following result.

Theorem 3 The function $\left.f\left(\bar{X}, z_{1}, z_{2}\right)\right|_{e_{1}, e_{2}}$ is concave over each sub-cell of $R\left(F_{C}\right)$, where cell $C$ belongs to the finite number of cells in the feasible region as formed in Section 3 and the sub-cells of $R\left(F_{C}\right)$ are formed by the ETTLs associated with $e_{1}$ and $C$, and $e_{2}$ and $C$.

Proof: Since the form of the distance function $r(\bar{X},(u, v))$ does not change as long as $\bar{X}$ is within a sub-cell of $R\left(F_{C}\right)$, a similar argument to that used in the proof of Lemma 4 allows us to conclude that $\left.f\left(\bar{X}, z_{1}, z_{2}\right)\right|_{e_{1}, e_{2}}$ is concave over each sub-cell of $R\left(F_{C}\right)$.

\section{Algorithm 2 (Single New Facility, Two Sub-aisles Case)}

1. FOR each cell $C \subset F$.

2. $\quad$ IF $F_{C} \neq \phi$ THEN

3. $\quad$ FOR each $R\left(F_{C}\right) \subset F_{C}$

4. $\quad$ FOR each edge $\left(u_{i}, v_{i}\right) \in A$

5. Generate the ETTL associated with $\left(u_{i}, v_{i}\right)$ and $C$

6. $\quad$ FOR each edge $\left(u_{j}, v_{j}\right) \in A(j \neq i)$

7. Generate the ETTL associated with $\left(u_{j}, v_{j}\right)$ and $C$

8. Let CORNER denote the set of corners associated with the sub-cells of $R\left(F_{C}\right)$

9. $\quad$ FOR each corner in CORNER

10. $\quad$ Find the shortest feasible rectilinear path from the corner to $\left(u_{i}, v_{i}\right)$

11. $\quad$ Find the shortest feasible rectilinear path from the corner to $\left(u_{j}, v_{j}\right)$

12. Evaluate the objective function and record the best solution 
The complexity analysis of Algorithms 1 and 2 is presented in Section 5. We briefly discuss the placement of multiple NFs. In this case, we need to consider the interactions between NFs and EFs as well as between NFs and NFs. A straightforward heuristic is to place one NF at one time sequentially, using Algorithm 1 or Algorithm 2.

\section{Solution Complexity}

It is clear that our solution method for the no sub-aisle situation has a finite number of steps. For the one sub-aisle case and two sub-aisles case, the number of cells is an important factor governing the solution complexity. Formally, $n$ rectangular EFs generate at most $3 n$ vertical gridlines and $3 n$ horizontal gridlines. Note that one gridline is generated from each EF associated with its single I/O point. Suppose that the original material handling network has $a_{v}$ vertical arcs and $a_{h}$ horizontal arcs. We use $a$ to signify the upper bound over $a_{v}$ and $a_{h}$, i.e. $a=\max \left\{a_{v}, a_{h}\right\}$. In the worst case $a$ additional vertical gridlines and $a$ additional horizontal gridlines are generated. Now we are ready to analyze the solution complexity.

The number of cells generated is at most $(3 n+a+1)^{2}$, therefore the number of sub-rectangles of feasible region is at most $3(3 n+a+1)^{2}$ The number of edges is at most $2 a(3 n+a)$. Accordingly, the number of edge pairs is bounded by $2 a^{2}(3 n+a)^{2}$. Therefore the solution complexity of Algorithm 1 in the worst case is $12 a(3 n+a+1)^{2}(3 n+a)$.

In addition, an edge pair will generate at most two ETTLs within a cell because each edge can generate at most one ETTL associated with this cell. Consequently, at most 9 corners (candidate placements) associated with a sub-rectangle of a cell are under consideration in Step 9 of Algorithm 2. As a result, the solution complexity of Algorithm 2 in the worst case is $54(3 n+a+1)^{2} a^{2}(3 n+a)^{2}$. Note that the above analysis does not include the complexity of finding feasible regions and the complexity of finding the shortest feasible rectilinear paths.

\section{Example}

This section illustrates our solution methodology with the aid of an example, depicted in Figure 14. The EFs, I/O locations, $h_{i j} \mathrm{~s}$ and $w_{i}$ s are shown in Figure 14. The bold lines in Figure 14 are the existing aisles. The dimensions of the NF and its I/O location can also be obtained from Figure 14.

Following the grid construction procedure, we draw several horizontal and vertical lines (dashed lines in Figure 14). Suppose that the unit construction cost $K=2$. First we identify the region for feasible placements, which is illustrated in Figure 15. As we can see, the feasible region is divided into a set of rectangular cells. There are 12 cells (labeled with "A" through "L") and 18 edges (labeled with "a" through "r") under consideration. For the case when no sub-aisle is constructed, 


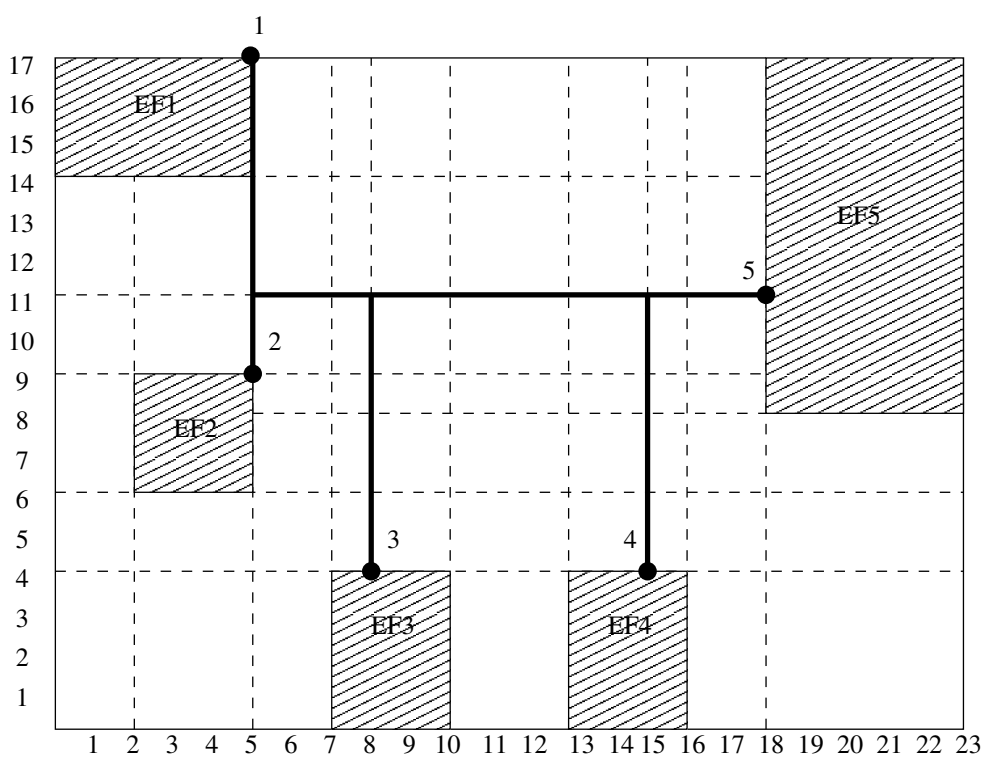

\begin{tabular}{|c|l|l|l|l|l|}
\multicolumn{7}{c|}{$\mathrm{h}_{\mathrm{ij}}$ values } \\
\hline${E F_{\mathrm{i}}}_{\mathrm{j}}$ & 1 & 2 & 3 & 4 & 5 \\
\hline 1 & & 4 & 0 & 2 & 1 \\
\hline 2 & & & 0 & 0 & 1 \\
\hline 3 & & & & 9 & 1 \\
\hline 4 & & & & & 2 \\
\hline 5 & & & & & \\
\hline
\end{tabular}

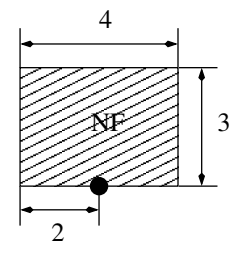

$\mathrm{w}_{\mathrm{i}}$ values

\begin{tabular}{|l|l|l|l|l|l|}
\hline $\mathrm{EF}_{\mathrm{i}}$ & 1 & 2 & 3 & 4 & 5 \\
\hline $\mathrm{w}_{\mathrm{i}}$ & 3 & 3 & 4 & 5 & 1 \\
\hline
\end{tabular}

Figure 14: Example.

there are 4 candidate placements and the corresponding results are shown in Table 1 . For the single sub-aisle case, there are a total of 38 candidate combinations as shown in Table 2. Many of these are dominated due to the established results. For the two sub-aisles case, the number of candidate combinations is very large. Only those candidate combinations that correspond to locations in cells $G$ and $H$ are shown in Table 3 . Note that $f_{1}$ and $f_{2}$ denote the material handling cost of NF-EF interactions and EF-EF interactions respectively, and $f_{3}$ accounts for the fixed construction cost of sub-aisles.

The optimal placement of the NF and the corresponding sub-aisle(s) construction with respect to the above three cases are illustrated in Figure 16. $\bar{X}_{1}, \bar{X}_{2}$ and $\bar{X}_{3}$ in Figure 16 correspond to the optimal placements, respectively and the dotted lines are the newly constructed sub-aisles. The optimal solutions for the three cases are listed in Table 4 from which we clearly observe that the two newly constructed aisles (one from $\bar{X}(10,4)$ to $(8,4)$ and another one from $\bar{X}(10,4)$ to $(15,4)$ ) significantly reduces the cost of EF-EF interactions from 338 to 212, and therefore this case achieves the minimum cost of 370 though accompanied with a higher cost of constructing two sub-aisles. 


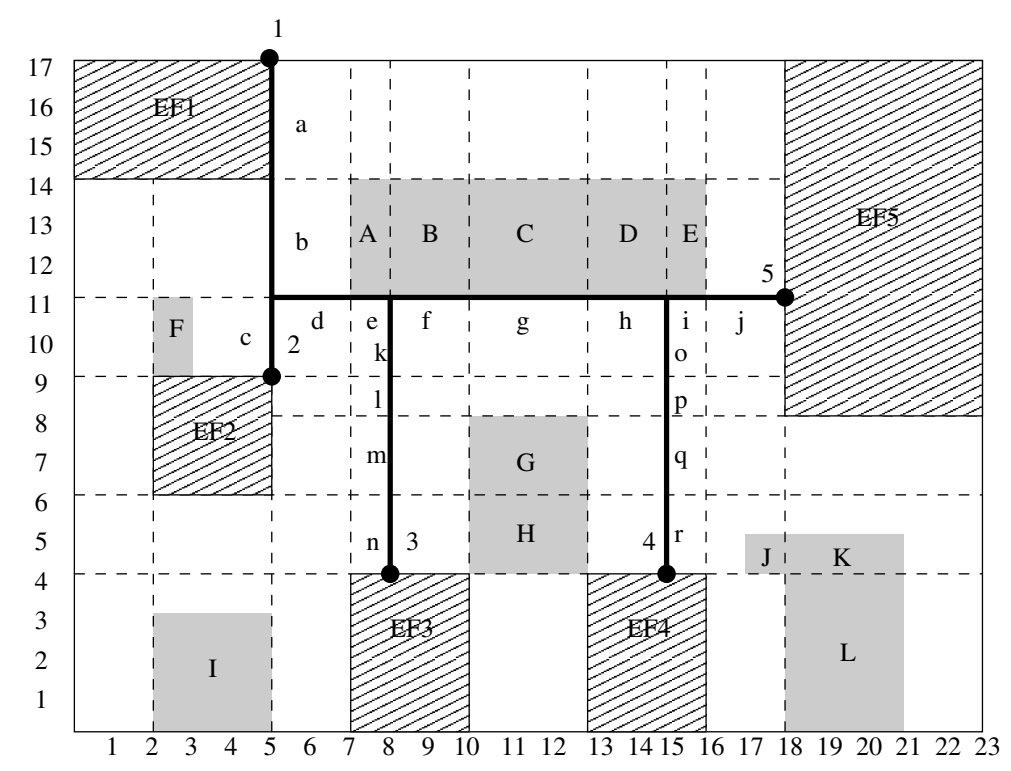

Figure 15: Feasible region for example.

Table 1: Results when no sub-aisle is constructed

\begin{tabular}{|cccc|}
\hline Case & $\bar{X}$ & $f_{1}+f_{2}$ & Remark \\
\hline 1 & $(7,11)$ & $154+338=492$ & \\
2 & $(8,11)$ & $150+338=488$ & Optimal \\
3 & $(15,11)$ & $178+338=516$ & \\
4 & $(16,11)$ & $192+338=530$ & \\
\hline
\end{tabular}

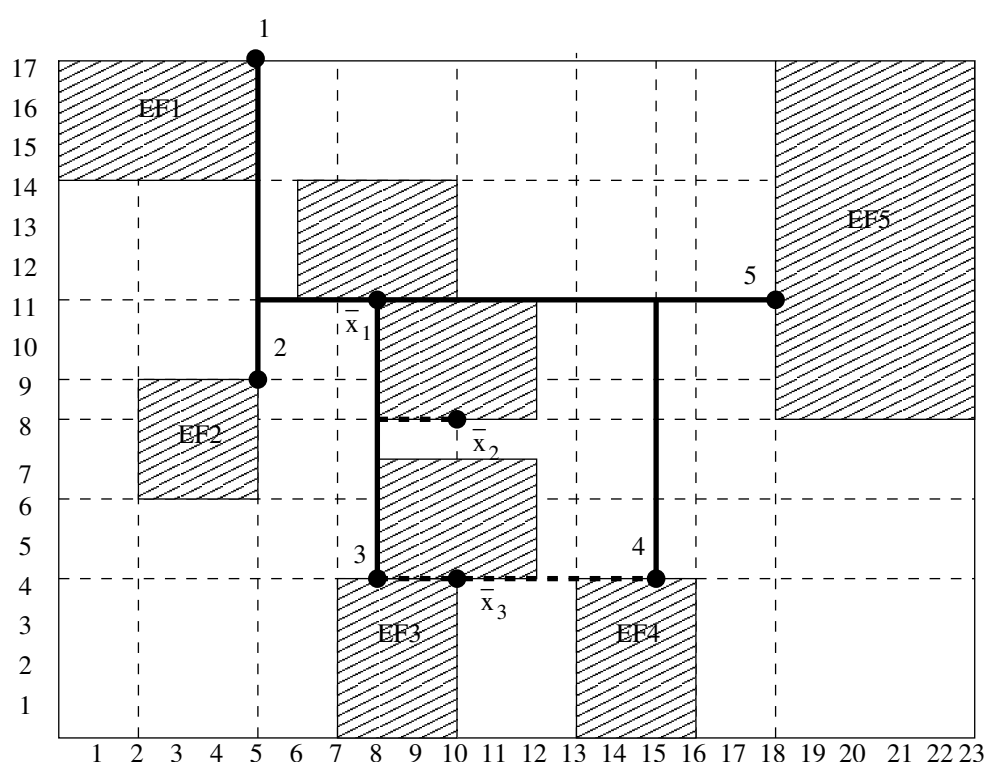

Figure 16: Solution for example. 
Table 2: Results when one sub-aisle is constructed

\begin{tabular}{|c|c|c|c|c|c|c|}
\hline Cell & Case & $\bar{X}$ & $z$ & $f_{1}+f_{2}$ & $f_{3}$ & Remark \\
\hline $\mathrm{A}$ & 1 & $(7,14)$ & $(5,14)$ & $224+338=562$ & 4 & \\
\hline B & 2 & $(8,14)$ & $(5,14)$ & & & dominated by case 1 \\
\hline $\mathrm{C}$ & 3 & $(10,14)$ & $(5,14)$ & & & dominated by case 1 \\
\hline $\mathrm{D}$ & 4 & $(13,14)$ & $(5,14)$ & & & dominated by case 1 \\
\hline $\mathrm{E}$ & 5 & $(15,14)$ & $(5,14)$ & & & dominated by case 1 \\
\hline $\mathrm{F}$ & 6 & $(3,11)$ & $(5,11)$ & $194+338=532$ & 4 & Optimal \\
\hline $\mathrm{F}$ & 7 & $(3,9)$ & $(5,9)$ & $214+338=552$ & 4 & \\
\hline $\mathrm{G}$ & 8 & $(10,8)$ & $(8,8)$ & $206+338=544$ & 4 & \\
\hline $\mathrm{G}$ & 9 & $(10,6)$ & $(8,6)$ & & & dominated by case 8 and 16 \\
\hline $\mathrm{G}$ & 10 & $(10,8)$ & $(12,11)$ & & & dominated by a non-aisle case \\
\hline $\mathrm{G}$ & 11 & $(13,8)$ & $(15,8)$ & $226+338=564$ & 4 & \\
\hline $\mathrm{G}$ & 12 & $(13,8)$ & $(11,11)$ & & & dominated by a non-aisle case \\
\hline $\mathrm{G}$ & 13 & $(13,6)$ & $(15,6)$ & & & dominated by case 11 and 19 \\
\hline $\mathrm{H}$ & 14 & $(10,6)$ & $(8,6)$ & & & dominated by case 8 and 16 \\
\hline $\mathrm{H}$ & 15 & $(10,6)$ & $(12,11)$ & & & dominated by case 10 \\
\hline $\mathrm{H}$ & 16 & $(10,4)$ & $(8,4)$ & $238+338=576$ & 4 & \\
\hline $\mathrm{H}$ & 17 & $(13,6)$ & $(15,6)$ & & & dominated by case 11 and 19 \\
\hline $\mathrm{H}$ & 18 & $(13,6)$ & $(11,11)$ & & & dominated by case 12 \\
\hline $\mathrm{H}$ & 19 & $(13,4)$ & $(15,4)$ & $250+338=588$ & 4 & \\
\hline I & 20 & $(5,3)$ & $(8,4)$ & & & dominated by case 16 \\
\hline I & 21 & $(5,3)$ & $(8,6)$ & & & dominated by case 9 \\
\hline I & 22 & $(5,3)$ & $(8,8)$ & & & dominated by case 8 \\
\hline I & 23 & $(5,3)$ & $(8,9)$ & $310+338=648$ & 18 & \\
\hline I & 24 & $(5,3)$ & $(7,11)$ & & & dominated by a non-aisle case \\
\hline I & 25 & $(5,3)$ & $(5,9)$ & $342+338=680$ & 20 & \\
\hline $\mathrm{J}$ & 26 & $(17,4)$ & $(15,4)$ & & & dominated by case 19 \\
\hline $\mathrm{J}$ & 27 & $(17,5)$ & $(15,5)$ & $246+338=584$ & 4 & \\
\hline $\mathrm{K}$ & 28 & $(18,4)$ & $(15,4)$ & & & dominated by case 26 \\
\hline $\mathrm{K}$ & 29 & $(18,5)$ & $(15,5)$ & & & dominated by case 27 \\
\hline $\mathrm{K}$ & 30 & $(18,5)$ & $(15,6)$ & & & dominated by case 13 \\
\hline $\mathrm{K}$ & 31 & $(18,5)$ & $(15,8)$ & & & dominated by case 11 \\
\hline $\mathrm{K}$ & 32 & $(18,5)$ & $(15,9)$ & $302+338=640$ & 14 & \\
\hline $\mathrm{K}$ & 33 & $(18,5)$ & $(16,11)$ & & & dominated by a non-aisle case \\
\hline $\mathrm{L}$ & 34 & $(18,4)$ & $(15,4)$ & & & dominated by case 26 \\
\hline $\mathrm{L}$ & 35 & $(18,4)$ & $(15,6)$ & & & dominated by case 13 \\
\hline $\mathrm{L}$ & 36 & $(18,4)$ & $(15,8)$ & & & dominated by case 11 \\
\hline $\mathrm{L}$ & 37 & $(18,4)$ & $(15,9)$ & & & dominated by case 32 \\
\hline $\mathrm{L}$ & 38 & $(18,4)$ & $(16,11)$ & & & dominated by case 33 \\
\hline
\end{tabular}


Table 3: Results when two sub-aisles are constructed

\begin{tabular}{|cccccccl|}
\hline Cell & Case & $\bar{X}$ & $z_{1}$ & $z_{2}$ & $f_{1}+f_{2}$ & $f_{3}$ & Remark \\
\hline $\mathrm{G}$ & 1 & $(10,8)$ & $(8,8)$ & $(12,11)$ & $182+338=520$ & 14 & \\
$\mathrm{G}$ & 2 & $(10,6)$ & $(8,6)$ & $(12,11)$ & $198+338=536$ & 18 & \\
$\mathrm{G}$ & 3 & $(10,8)$ & $(8,8)$ & $(15,8)$ & $152+277=429$ & 14 & \\
$\mathrm{G}$ & 4 & $(10,6)$ & $(8,6)$ & $(15,6)$ & $148+248=396$ & 14 & \\
$\mathrm{G}$ & 5 & $(13,8)$ & $(15,8)$ & $(11,11)$ & $188+338=526$ & 14 & \\
$\mathrm{G}$ & 6 & $(13,6)$ & $(15,6)$ & $(11,11)$ & $200+338=538$ & 18 & \\
$\mathrm{G}$ & 7 & $(13,8)$ & $(15,8)$ & $(8,8)$ & $164+284=429$ & 14 & \\
$\mathrm{G}$ & 8 & $(13,6)$ & $(15,6)$ & $(8,6)$ & $160+248=396$ & 14 & \\
$\mathrm{H}$ & 9 & $(10,6)$ & $(8,6)$ & $(12,11)$ & & & dominated by case 2 \\
$\mathrm{H}$ & 10 & $(10,6)$ & $(8,6)$ & $(15,6)$ & & & dominated by case 4 \\
$\mathrm{H}$ & 11 & $(10,4)$ & $(8,4)$ & $(12,11)$ & $214+338=552$ & 22 & \\
$\mathrm{H}$ & 12 & $(10,4)$ & $(8,4)$ & $(15,4)$ & $144+212=356$ & 14 & Optimal \\
$\mathrm{H}$ & 13 & $(13,6)$ & $(15,6)$ & $(11,11)$ & & & dominated by case 6 \\
$\mathrm{H}$ & 14 & $(13,4)$ & $(15,4)$ & $(11,11)$ & $212+338=550$ & 22 & \\
$\mathrm{H}$ & 15 & $(13,6)$ & $(15,6)$ & $(8,6)$ & & & dominated by case 8 \\
$\mathrm{H}$ & 16 & $(13,4)$ & $(15,4)$ & $(8,4)$ & $156+212=356$ & 14 & \\
\hline
\end{tabular}

Table 4: Effect of the number of constructed sub-aisles

\begin{tabular}{|ccccc|}
\hline \# of sub-aisles & $\bar{X}$ & $z_{1}$ & $z_{2}$ & $f_{1}+f_{2}+f_{3}$ \\
\hline 0 & $(8,11)$ & $/$ & $/$ & $150+338+0=488$ \\
1 & $(3,11)$ & $(5,11)$ & $/$ & $194+338+4=536$ \\
2 & $(10,4)$ & $(8,4)$ & $(15,4)$ & $144+212+14=370$ \\
\hline
\end{tabular}




\section{Summary and Conclusions}

The paper has addressed the layout addition problem of placing a rectangular NF in a plane in the presence of other rectangular EFs and an aisle structure. The major distinctive feature of our work is that we study this problem under the restriction that travel occurs only on aisles. A specific contribution is that we integrate placement of the NF and configuration of one or two sub-aisles which connect the NF to the existing aisle structure. The motivation for our modeling framework is that material handling is normally restricted to aisles.

We show that there are a finite number of candidate locations when we need to place the NF directly on to the aisle structure. When locating the NF off the existing aisles, we show that the optimal configuration of a sub-aisle has to be a shortest feasible rectilinear path between the location of the NF and the edge to which the NF is connected. We also show that there are a finite number of candidate locations for the NF in the cases of constructing one or two sub-aisles. We present algorithms for various variations of the problem, and study their complexity. Finally, an example is presented to investigate the impact of the number of sub-aisles added.

Our conclusions are as follows: The no sub-aisle and single sub-aisle cases can be analyzed using similar tools to that of the $p$-median problem on a network, and results in the identification of a finite number of candidate placements for the NF and a finite number of corresponding subaisle configurations. The two sub-aisles case is different because the addition of two sub-aisles can impact travel distance between the EFs as well. Its analysis is more complex, but the basic result of a finite number of candidate locations for the NF and configurations for the sub-aisles remains unchanged. A final conclusion is that adding two sub-aisles results in significant benefits.

\section{Acknowledgement}

This work gratefully acknowledges support from the National Science Foundation, via grant DMI0300370. The authors are also grateful to the efforts of a dedicated referee whose comments have substantially improved the exposition of this paper.

\section{References}

Aneja, Y. P. and M. Parlar (1994). Algorithms for weber facility location in the presence of forbidden regions and/or barriers to travel. Transportation Science 28(1), 70-76.

Batta, R., A. Ghose, and U. Palekar (1989). Locating facilities on the manhattan metric with arbitrarily shaped barriers and convex forbidden regions. Transportation Science 23(1), 2636. 
Butt, S. E. and T. M. Cavalier (1996). An efficient algorithm for facility location in the presence of forbidden regions. European Journal of Operational Research 90(1), 56-70.

Church, R. L. and C. ReVelle (1979). Location modeling utilizing maximum service distance criteria. Geographical Analysis 11, 358-373.

Dearing, P. M., H. W. Hamacher, and K. Klamroth (2002). Dominating sets for rectilinear center location problems with polyhedral barriers. Naval Research Logistics 49(7), 647-665.

Drezner, Z. and H. W. Hamacher (2004). Facility Location: Applications and Theory. NY, Heidelberg: Springer-Verlag.

Francis, R. L., L. F. McGinnis Jr., and J. A. White (1992). Facility Layout and Location: An Analytical Approach. NJ, Upper Saddle River: Prentice Hall.

Hakimi, S. L. (1964). Optimum locations of switching centers and the absolute centers and medians of a graph. Operations Research 12(3), 450-459.

Katz, I. N. and L. Cooper (1981). Facility location in the presence of forbidden regions, I: formulation and the case of euclidean distance with one forbidden circle. European Journal of Operational Research 6(2), 166-173.

Kelachankuttu, H., R. Batta, and R. Nagi (2007). Contour line construction for a new rectangular facility in an existing layout with rectangular departments. European Journal of Operational Research 180(1), 149-162.

Klamroth, K. (2000). Single facility location problems with barriers. Habilitation thesis, University of Kaiserslautern, Germany.

Kuehn, A. A. and M. J. Hamburger (1963). A heuristic program for locating warehouses. Management Science 9(4), 643-666.

Larson, R. and V. Li (1981). Finding minimum rectilinear distance paths in the presence of barriers. Networks 11 (3), 285-304.

Larson, R. C. and G. Sadiq (1983). Facility locations with the manhattan metric in the presence of barriers to travel. Operations Research 31(4), 652-669.

Melkote, S. (1996). Integrated models of facility location and network design. Ph. D. thesis, Northwestern University.

Nandikonda, P., R. Batta, and R. Nagi (2003). Locating a 1-center on a manhattan plane with "arbitrarily" shaped barriers. Annals of Operations Research 123, 157-172.

Sarkar, A., R. Batta, and R. Nagi (2005). Planar area location/layout problem in the presence of generalized congested regions with the rectilinear distance metric. IIE Transactions 37(1), $35-50$. 
Sarkar, A., R. Batta, and R. Nagi (2007). Placing a finite size facility with a center objective on a rectilinear plane with barriers. European Journal of Operational Research 179(3), 1160-1176.

Savas, S., R. Batta, and R. Nagi (2002). Finite-size facility placement in the presence of barriers to rectilinear travel. Operations Research 50(6), 1018-1031.

Toregas, C., R. Swain, and L. Bergmann (1971). The location of emergency service facilities. Operations Research 19(6), 1363-1373. 